\title{
Editorial: Chemical Ecology and Conservation Biological Control
}

\author{
Stefano Colazza ${ }^{1 *}$, Maria L. Pappas ${ }^{2}$, Anne Marie Cortesero ${ }^{3}$ and \\ Cesar Rodriguez-Saona ${ }^{4}$ \\ ${ }^{1}$ Department of Agricultural, Food and Forest Sciences, University of Palermo, Palermo, Italy, ${ }^{2}$ Laboratory of Agricultural \\ Entomology and Zoology, Department of Agricultural Development, Democritus University of Thrace, Orestiada, Greece, \\ ${ }^{3}$ IGEPP-UMR 1349, INRAE, Institut Agro, Univ Rennes 1, Rennes, France, ${ }^{4}$ Department of Entomology, Rutgers, The State \\ University of New Jersey, New Brunswick, NJ, United States
}

Keywords: semiochemicals, natural enemies, herbivore-induced plant volatiles, methyl salicylate, agriculture

Editorial on the Research Topic

Chemical Ecology and Conservation Biological Control

Conservation Biological Control (CBC) in agroecosystems requires a landscape management perspective based on a set of tactics and approaches to preserve and improve the efficacy of natural enemies to control the populations of insect pests (Landis et al., 2000; Tscharntke et al., 2007; Shields et al., 2019). In recent years, extensive research has elucidated the key role of chemical ecology in the multitrophic interactions among plants, arthropod herbivores, and their natural enemies, and revealed the potential of chemical cues to manipulate natural enemies in CBC programs (Khan et al., 2008; Colazza et al., 2017). Plants have evolved a plethora of chemical compounds to mediate the multitrophic complex interactions among the members of the plant-associated arthropod community. Plant defense responses are categorized as constitutive, always present in the plant, or induced, produced in response to biotic stresses (Pappas et al., 2017). In the latter case, plants attacked by herbivores emit a variety of chemical compounds, which can protect them directly by targeting the herbivores via their toxic properties or by being repellent, or indirectly, by attracting arthropod natural enemies of such herbivores (Pappas et al., 2017). To locate their host or prey, natural enemies encounter and explore a variety of stimuli, among which the chemical cues, named semiochemicals, i.e. insect-produced kairomones and herbivore-induced plant volatiles (HIPVs), play a relevant role to attract them into the agroecosystems (Kaiser et al., 2017; Turlings and Erb, 2018). Over the last two decades, research on exploiting chemical ecology in CBC has provided new insight on the attraction of natural enemies mediated by semiochemicals (Rodriguez-Saona et al., 2012; Peri et al., 2018). In this Research Topic, the editors present one review article and six research articles from ten countries in Europe, Africa, North America, and Asia that discuss how semiochemicals could be applied in CBC, where the use of insecticides needs to be decreased.

In a review article, Ayelo et al. provide extensive examples of field application of semiochemicals, in particular kairomones (e.g., HIPVs or insect-produced chemicals such as pheromones), in CBC to increase the number of natural enemies attracted to a crop. The authors argue the need for future field studies to focus on the application of kairomone blends rather than single kairomones, which currently dominate the literature on field attractants for natural enemies. They discuss potential strategies for improving kairomone use through attract-and-reward techniques, olfactory associative learning, and optimization of kairomone lure formulations. The authors conclude by debating that the effectiveness of kairomone use for enhancing CBC strategies should move from demonstration of increase in the number of attracted natural enemies to reducing pest populations and crop damage below economic threshold levels and increasing crop yield. 
Egg deposition on plant organs is usually the first step of an herbivorous insect attack. In the last years, numerous studies made evident the response of the plants to insect oviposition by the emission of plant volatiles, namely oviposition-induced plant volatiles (OIPVs), which can recruit egg parasitoids of the attacking herbivore, and provide, in this way, an effective defense against insect pests (Fatouros et al., 2016). Recent studies on the Brassicaceae family have shown that plants can also apply direct defense mechanisms against the eggs of specialist Pieridae butterflies by developing a hypersensitive response (HR)-like leaf necrosis which is able to detach the eggs from the plant surface, causing their falling to the ground (Fatouros et al., 2015). The original research article by Afentoulis et al. showed that crop plants within the Brassicaceae family possess such so called "eggkilling" traits and can exert the double-defense line, which may enable effective selection of egg-killing defense traits by cabbage breeders. The authors evaluated the responses of different lines of the egg parasitoid Trichogramma evanescens to OIPVs emitted by Brassica nigra and three close relatives, $B$. napus, B. rapa, and $B$. oleracea. The results showed intraspecific variation in the female wasps' response to OIPVs during host searching, which should be taken into account when selecting parasitoid lines for use in the biocontrol of pests in certain crops.

Synthetic HIPVs are thought to provide the best potential for developing effective and practical semiochemical-based strategies for manipulating parasitoid populations. Methyl salicylate (MeSA) is an HIPV shown to have biological relevance for carnivorous arthropod attraction (Rodriguez-Saona et al., 2011). In this collection, Lee et al. investigated whether the application of lures with synthetic MeSA influences the activities of natural enemies in the field. Experiments conducted on spruce fields recorded fewer aphids and more aphid parasitoids when baited with MeSA compared to the control fields. Similar results were observed in experiments conducted in red maple fields, as sticky cards and pan traps in MeSA-treated plots captured more lady beetles and predatory mirids. Green leaf volatiles (GLV) are usually the first compounds emitted by plants in response to herbivore damage, whereas MeSA follow later and is still detectable from plants hours or days later (Turlings and Erb, 2018). GLVs are involved in the regulation of plant defense genes and defense priming in different plant species as well as in the recruitment of insect parasitoids (Engelberth et al., 2004). McPike and Evenden research article tested the attractiveness and retention of MeSA and two GLVs, (Z)-3-hexenol and (Z)3-hexenyl-acetate, on the parasitoid Apanteles polychrosidis in ash trees infested by Caloptilia fraxinella. The response of the

\section{REFERENCES}

Colazza, S., Shields, M. W., Peri, E., and Cusumano, A. (2017). Ecosystem services provided by unmanaged habitats in agricultural landscapes. Environmental Pest Management: Challenges for Agronomists, Ecologists, Economists and Policymakers. 153 doi: 10.1002/9781119255574.ch7

Engelberth, J., Alborn, H. T., Schmelz, E. A., and Tumlinson, J. H. (2004). Airborne signals prime plants against insect herbivore attack. PNAS. 101, 1781-1785. doi: $10.1073 /$ pnas. 0308037100 parasitoid to these same compounds was also evaluated in the laboratory using olfactometer studies. Two original papers investigated the role of HIPVs in field experiments. Riahi et al. showed that individual exposure of sweet pepper plants to $(Z)$ 3-hexenol, $(Z)$-3-hexenyl acetate, $(Z)$-3-hexenyl propanoate, $(Z)$ 3-hexenyl butanoate, and MeSA activates defensive signaling pathways. Uefune et al. reported that dispensers lured with a blend of volatile comprising ( $Z$ )-3-hexenyl acetate, $\alpha$-pinene, sabinene, and $n$-heptanal attract Cotesia vestalis, a specialist parasitoid wasp of diamondback moth larvae.

HIPVs may guide host searching parasitoids at long range, while chemicals of low volatility may become important for host location at a short range (Vet and Dicke, 1992). Recently, it was demonstrated that chemical trails left by host insects on the plant surface or feeding substrate consist of low volatility cuticular hydrocarbons (CHCs), which could be exploited by female parasitoids as contact kairomones. The original research article by Arif et al. provides behavioral and chemical evidence on the role of specific cuticular compounds left on the substrate by Halyomorpha halys, which are subsequently exploited as contact chemical cues by its egg parasitoid Trissolcus japonicus while foraging for host eggs. Female wasps explored more intensively and spent more time on female-host-associated chemical traces over male-host-associated ones. Furthermore, the authors identified the linear hydrocarbon 1-hexadecene, the putative component in determining these differences, being more abundant in host males. In fact, hexane extracts of $H$. halys females blended with synthetic 1-hexadecene significantly reduced the wasps' arrestment responses compared to crude extracts.

In summary, the collection of articles in this Research Topic demonstrates that semiochemicals act in various aspects of insect-plant interactions at multiple levels, and highlights their importance for developing efficient CBC programs.

\section{AUTHOR CONTRIBUTIONS}

All authors listed have made a substantial, direct, and intellectual contribution to the work and approved it for publication.

\section{ACKNOWLEDGMENTS}

We would like to take this opportunity to thank the contributions of the authors and the Journal Specialists for their consistent technical support. 
Kaiser, L., Ode, P., van Nouhuys, S., Calatayud, P. A., Colazza, S., Cortesero, A. M., Thiel, A., and van Baaren, J. (2017). The plant as a habitat for entomophagous insects. Adv. Bot. Res. 81, 179-223. doi: 10.1016/bs.abr.2016.09.006

Khan, Z. R., James, D. G., Midega, C. A., and Pickett, J. A. (2008). Chemical ecology and conservation biological control. Biol. Control. 45, 210-224. doi: 10.1016/j.biocontrol.2007.11.009

Landis, D. A., Wratten, S. D., and Gurr, G. M. (2000). Habitat management to conserve natural enemies of arthropod pests in agriculture. Annu. Rev. Entomol. 45, 175-201 doi: 10.1146/annurev.ento.45.1.175

Pappas, M. L., Broekgaarden, C., Broufas, G. D., Kant, M. R., Messelink, G. J., Steppuhn, A., Wäckers, F., and Van Dam, N. M. (2017). Induced plant defences in biological control of arthropod pests: a double-edged sword. Pest Management Science. 73, 1780-1788. doi: 10.1002/ps.4587

Peri, E., Moujahed, R., Wajnberg, E., and Colazza, S. (2018). Applied chemical ecology to enhance insect parasitoid efficacy in the biological control of crop pests. In Chemical Ecology of Insects. CRC Press. p. 234-267. doi: 10.1201/9781351228398-10

Rodriguez-Saona, C., Blaauw, B., and Isaacs, R. (2012). "Manipulation of natural enemies in agroecosystems: habitat and semiochemicals for sustainable insect pest control," in Integrated Pest Management and Pest Control - Current and Future Tactics, Larramendy, M. L. and Soloneski, S., eds (Rijeka, Croatia: IntechOpen) 89-126. doi: 10.5772/30375

Rodriguez-Saona, C., Kaplan, I., Braasch, J., Chinnasamy, D., and Williams, L. (2011). Field responses of predaceous arthropods to methyl salicylate: a meta-analysis and case study in cranberries. Biol. Control. 59, 294-303. doi: 10.1016/j.biocontrol.2011.06.017

Shields, M. W., Johnson, A. C., Pandey, S., Cullen, R., González-Chang, M., et al. (2019). History, current situation and challenges for conservation biological control. Biol. Control. 131, 25-35. doi: 10.1016/j.biocontrol.2018. 12.010
Tscharntke, T., Bommarco, R., Clough, Y., Crist, T. O., Kleijn, D., Rand, T. A., Tylianakis, J. M., van Nouhuys, S., and Vidal, S. (2007). Conservation biological control and enemy diversity on a landscape scale. Biol. Control. 43, 294-309. doi: 10.1016/j.biocontrol.2007.08.006

Turlings, T. C. J., and Erb, M. (2018). Tritrophic interactions mediated by herbivore-induced plant volatiles: mechanisms, ecological relevance, and application potential. Annu. Rev. Entomol. 63, 433-452. doi: 10.1146/annurev-ento-020117-043507

Vet, L. E. M., and Dicke, M. (1992). Ecology of infochemical use by natural enemies in a tritrophic context. Ann. Rev. Entomol. 37, 141-172. doi: 10.1146/annurev.en.37.010192.001041

Conflict of Interest: The authors declare that the research was conducted in the absence of any commercial or financial relationships that could be construed as a potential conflict of interest.

Publisher's Note: All claims expressed in this article are solely those of the authors and do not necessarily represent those of their affiliated organizations, or those of the publisher, the editors and the reviewers. Any product that may be evaluated in this article, or claim that may be made by its manufacturer, is not guaranteed or endorsed by the publisher.

Copyright (®) 2022 Colazza, Pappas, Cortesero and Rodriguez-Saona. This is an open-access article distributed under the terms of the Creative Commons Attribution License (CC BY). The use, distribution or reproduction in other forums is permitted, provided the original author $(s)$ and the copyright owner(s) are credited and that the original publication in this journal is cited, in accordance with accepted academic practice. No use, distribution or reproduction is permitted which does not comply with these terms. 